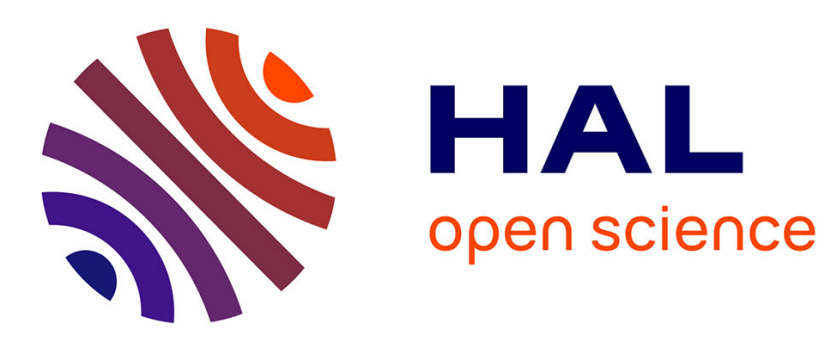

\title{
Damaged digital surfaces also deserve realistic healing
} Arthur Francisco, Noël Brunetière, Gildas Merceron

\section{To cite this version:}

Arthur Francisco, Noël Brunetière, Gildas Merceron. Damaged digital surfaces also deserve realistic healing. Surface Topography: Metrology and Properties, 2020, 8 (3), pp.035008. 10.1088/2051672X/aba7a3 . hal-03042203

\section{HAL Id: hal-03042203 \\ https://hal.science/hal-03042203}

Submitted on 6 Dec 2020

HAL is a multi-disciplinary open access archive for the deposit and dissemination of scientific research documents, whether they are published or not. The documents may come from teaching and research institutions in France or abroad, or from public or private research centers.
L'archive ouverte pluridisciplinaire HAL, est destinée au dépôt et à la diffusion de documents scientifiques de niveau recherche, publiés ou non, émanant des établissements d'enseignement et de recherche français ou étrangers, des laboratoires publics ou privés. 


\title{
Damaged Digital Surfaces Also Deserve Realistic Healing
}

\author{
Arthur Francisco, Noël Brunetière and Gildas Merceron \\ Institut Pprime, CNRS, Université de Poitiers, ISAE-ENSMA, F-86962 Futuroscope \\ Chasseneuil, France \\ E-mail: arthur.francisco@univ-poitiers.fr \\ April 2020
}

\begin{abstract}
Modern image processing software include inpainting tools for various applications: removing an undesirable feature from an image, restoring damaged parts of old photographs, erasing watermarks, etc. But as concern digital surfaces, the only way of erasing remaining dirt or filling in unread height zones is to apply Laplace's operator. Laplace's operator has many advantages: it is easy to implement, it minimizes the surface curvature i.e. no particular relief is created, it is only defined by the damaged zone frontier and the result is the same whatever the software. The major drawback is its excessive smoothness: when the hole to be fill in is large compared to the correlation length, a Laplacian patch contrasts too much with the neighboring relief. When dealing with digital surface study, the problem is not as much the visual aspect of the healing as its potential consequences on the surface parameters. Three alternative healing methods are applied on rough surfaces: a totally new approach, and pointand exemplar-based methods. The accuracy of the repairs are compared by means of parametric and statistical analyses. The results show that, unlike any expectation, Laplacian healing is efficient for small repairs but that point- and exemplar-based methods are statistically better solutions.
\end{abstract}

Keywords: inpainting, surface healing, dental micro-wear

\section{Introduction}

Image inpainting consists in filling in damaged areas or missing value areas in an image. It is a well-known process in the field of image processing. The aim of image inpainting was originally to restore damaged photographs by removing pits and scratches. The main difficulty in filling in spaces is to obtain a realistic result; ideally speaking the correction should not be apparent. When the damaged areas are small enough, simple tools like Laplace's correction succeed in healing the image. The repair can be compared to a skin stretched over the hole border. Healing a picture in such a way can be satisfactory in low color gradient areas, but as soon as the restored zone crosses a contrasted feature, the result becomes too apparent. It is the key point that has initiated 
the present study: real worn surfaces — from mechanical components for instance exhibit most of the time roughness, making Laplace's restoration not fitted for image healing needs; at least, at first sight. It is the only available tool in digital surface software, though.

There are many inpainting methods devoted to picture restoration: some are common-sense processes but other involve modern techniques like AI, artificial intelligence, with impressive results [1]. Let's put aside the latter, because they take advantage of complex neural networks that are trained to recreate special features like realistic eyes on a face, windows on a building, etc. By contrast, the need in digital surface restoration is just filling in areas by reproducing the neighboring texture. It is a more affordable task, for which widely used methods like point- and exemplar-based methods are well suited. There is an abundant literature on inpainting technology, presenting the historical needs for that kind of tools in image and video processing. Instead of collecting an exhaustive list of papers on the subject, one may refer to synthetic literature reviews as in Harrison thesis [2], Gong et al. book [3], chapter six and Barbu's book [4] for mathematical-based methods. As mentioned above, we choose to restrict the investigation field to simple algorithms, as the aim of the present work is to prove that affordable alternatives to Laplace's healing are worth to be used for digital surfaces.

Bertalmio et al. [5] fill in a hole, smoothly propagating the isophote lines - lines of equal color level - inward, using the surrounding existing points. The outside isophote are determined and extended inside the hole using the discretized Laplacian. Despite the major drawback of not reproducing the image texture - the short wavelengths this paper has been a starting point for many other works. A few years later, Bertalmio et al. [6] update, and signigicantly improve, the technique using image decomposition: structure and texture. The structure is the macro features, and the texture includes the short wavelength features. The structure restoration is performed following their first work, and the texture creation is ensured according Efros and Leung's seminal paper [?]. Efros and Leung's algorithm works well for textured images, where texture refers to pattern - texels - rather than various short wavelength features. Note that noisy compounds are preserved as well because unlike the preceding approach, the new points are created by copying points of the surroundings. For this purpose, a few known neighboring points of a centered point to be created are compared to the existing ones outside the hole: when a matching set of points is found, the central point is copied. The process goes on until there is no remaining point to be filled. A refined and efficient method is proposed by Harrison [7] and improved later [2]. Bertalmio's work is based on Partial Differential Equations, Efros' and Harrison's works are based on pixel surroundings, and defined here as point-based. Another way of reproducing information inside a hole, taking into account similar zones outside, is to copy several contiguous pixels at a time. As for point-based techniques, a metric is defined to compare an inside kernel to an outside target kernel; when they match, the inside pixels are replaced by a copy of the outside pixels. Criminisi et al. [8] use this strategy, along with isophote 
determination, to propagate patterns and preserve high frequencies. The approach, called exemplar-based, also produces very convincing results in removing objects in a picture.

The last two approaches - point- and exemplar-based methods - seem well indicated

for a digital surface restoration. Where picture information is made of three color layers, digital surface information is heights. It makes no big difference in the algorithm implementation. However, unlike image inpainting goal, which is only visual, digital surface healing must not significantly alter the subsequent analyzes to carry out. In the present work, we introduce a new approach which aims to preserve the surface anisotropy as well as the height statistics. It is referred as global method. To test the efficiency of the different inpainting methods, three surfaces are especially selected. They are chosen among dental surfaces, because they are sometimes subject to artifacts: in most cases, the artifacts are due to sharp slopes but it can also be due to remaining bubbles in the mold. The surfaces have different anisotropy ratios, and different height statistics. Many parameters are calculated on both original surfaces and arbitrarily damaged then repaired surfaces. If the results remain close for a particular method, it is considered as efficient; the visual result criterion alone is no more sufficient.

\section{Material}

As stated before, dental surfaces exhibit a great variability in roughness levels, in feature distribution, etc. To accurately catch the micro-texture of teeth, the dental surfaces are molded with a silicon polyvinylsiloxane elastomer. Then, the mold surfaces are digitized with a confocal, white light profilometer (Leica Microsystem DCM8; 100× magnification

lens, Numerical Aperture $=0.9$; Working Distance $=0.9 \mathrm{~mm}$, Lateral Resolution up to $140 \mathrm{~nm}$, Vertical Resolution up to $2 \mathrm{~nm}$ ).

Three surfaces are chosen for their anisotropy differences. The surfaces have their $8^{\text {th }}$ degree least square polynomial subtracted, so that the long wavelength texture is removed [9], [10].

\section{Anisotropy1 surface}

Anisotropy1 is a dental surface from a semi-wild cattle Bos taurus from Camargue, Rhône delta, France. Because it feeds on high amounts of herbaceous dicots, a strong anisotropy is observed, see figure 1. 


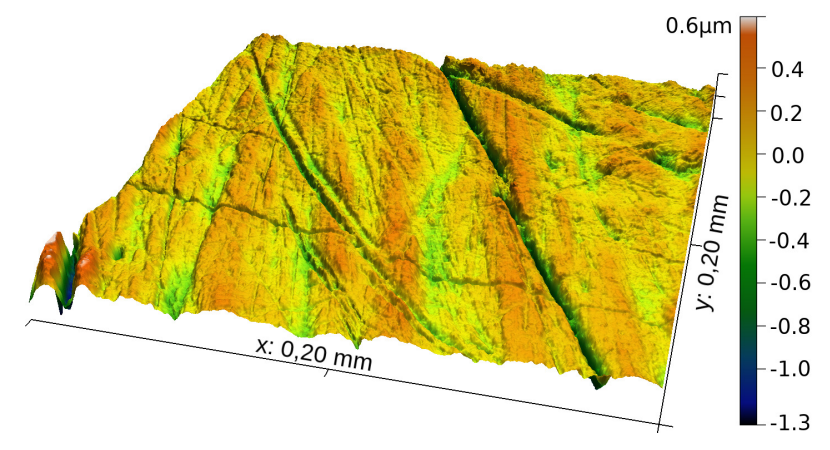

Figure 1: Bos taurus, anisotropy: high

Anisotropy2 surface

Anisotropy2 is a dental surface from the fruit-dominant browser yellow-backed duiker Cephalophus silvicultor. Figure 2 exhibits longer wavelengths than Anisotropy1, but the anisotropy factor is smaller.

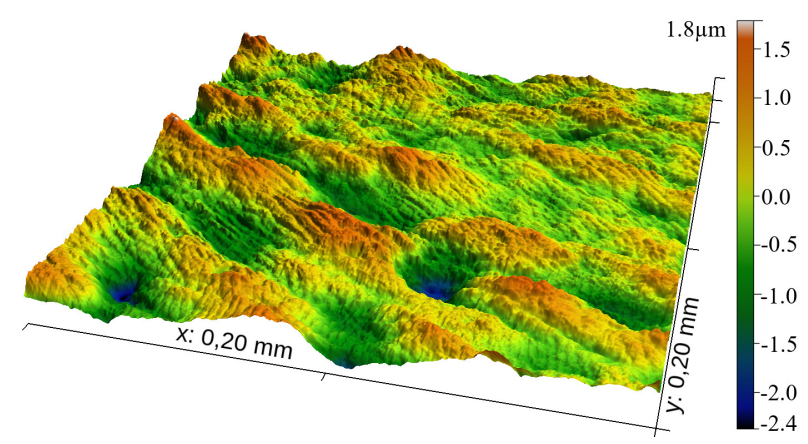

Figure 2: Cephalophus silvicultor, anisotropy: moderate

Anisotropy3 surface

Anisotropy3 is a dental surface from a red deer Cervus elaphus from Białowieża, Poland which feeds on grass, forbs and fruits. It can be seen on figure 3 that there is no preferred texture direction, thus the surface is globally isotropic. 


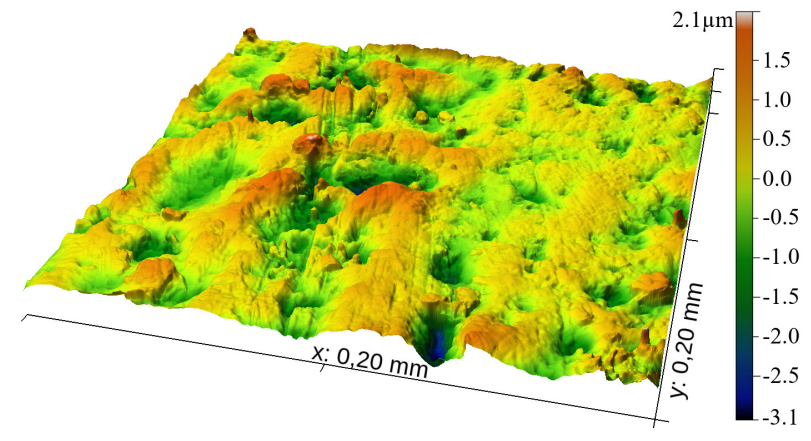

Figure 3: Cervus elaphus, anisotropy: low

\section{Methods}

First of all, Laplace's restoration is so often used that we shall explain why. The principle is to minimize the surface of the patch given the border heights.

\subsection{Laplacian filling}

When 1D data are to be filled between two points with no particular assumption, linear interpolation is used. However, regarding 2D data inside a closed curve - a "hole" border - linear interpolation is no longer applicable, see figure 4 for an illustration. The lesser constraint becomes the so-called "minimal surface"; to put it simple each point minimizes the surface created by its neighboring points.

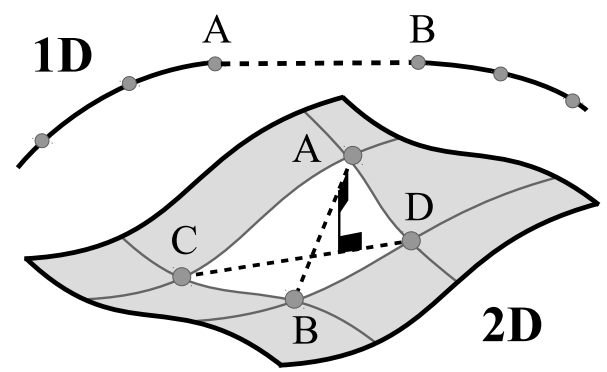

Figure 4: The points $A, B, C$ and $D$ are not coplanar, $(A B)$ and $(C D)$ do not intersect. There is no reason to linearly interpolate $[A B]$ rather than $[C D]$ : if a central point is to be drawn, it is at a fraction of the distance between $(A B)$ and $(C D)$

A mathematical explanation for the use of the Laplacian can be found in the Electronic Supplementary Material (ESM0). Another way of seeing things is to consider that each point inside the hole is the mean of its closest neighboring points. Let $\Omega$ be uniformly discretized and $f_{i, j}$ be the surface height at point $(i, j)$ on the grid. For the sake of simplicity, the grid step is supposed to be the same along $x$ and $y: h_{x}=h_{y}=h$. 
The simplest way of expressing that $f_{i, j}$ is the mean of the closest points is:

$$
f_{i, j}=\frac{1}{4}\left(f_{i, j-1}+f_{i-1, j}+f_{i, j+1}+f_{i+1, j}\right)
$$

or,

$$
f_{i, j-1}+f_{i-1, j}+f_{i, j+1}+f_{i+1, j}-4 f_{i, j}=0
$$

Equation 1 is also the discretized Laplacian applied to $f$. Indeed, discretizing $\partial^{2} f / \partial x^{2}$ using centered finite differences yields:

$$
\left(\frac{\partial^{2} f}{\partial x^{2}}\right)_{i, j}=\frac{1}{h}\left(\frac{f_{i+1, j}-f_{i, j}}{h}-\frac{f_{i, j}-f_{i-1, j}}{h}\right)
$$

and then,

$$
(\Delta f)_{i, j}=\frac{1}{h^{2}}\left(f_{i, j-1}+f_{i-1, j}+f_{i, j+1}+f_{i+1, j}-4 f_{i, j}\right)
$$

The most valuable qualities of Laplacian healing are its processing speed and robustness. The later means that no "surprising" data are introduced, whatever the hole shape, because the patch is extremely smooth: it is a soap film lying on the hole boundary, figure 5 .

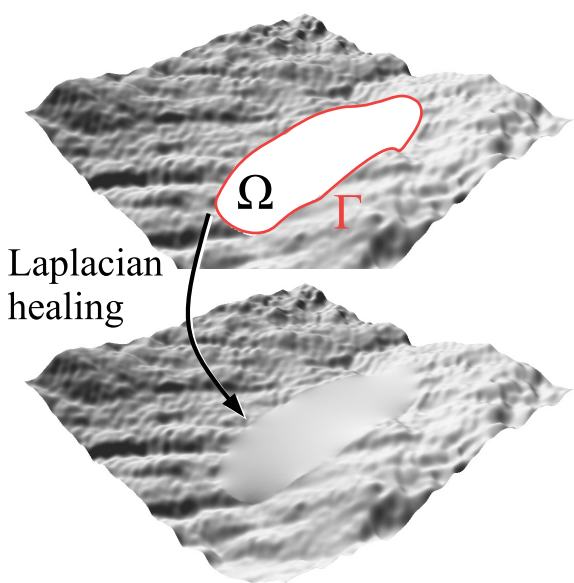

Figure 5: The Laplacian operator fills in $\Omega$ from the hole frontier $\Gamma$. The shape that is obtained is the same as the one of a soap film lying on the frontier

The main drawback of the Laplacian healing is obviously the other side of the minimal surface principle. When the hole is large compared to the apparent wavelength, the patch becomes too much apparent, and may alter the surface parameters, such as the height statistics. Because it does not take into account the hole vicinity, the more complex the surface is, the poorer the result. However, Laplace's interpolation is well suited for small holes, even if they account for an important ratio of missing data, provided that they are well scattered over the surface. 


\subsection{Global approach inpainting}

The goal is to propose a novel technique to fill in surface holes taking into account the surface overall anisotropy and the height statistics of the hole vicinity. It is an ad hoc technique that aims to reproduce the global characteristics of the surface inside the hole.

The main idea is to:

Step 1: fill in the hole area using Laplacian interpolation

Step 2: remove the fifth order least square polynomial of the surface masking the hole area

Step 3: determine a convenient digital filter $H$, and then apply it to the surface heights

Step 4: determine the height profile of the crown and generate as many heights as needed and fill in the hole area

Step 5: reintroduce the polynomial

As a result the anisotropy inside the hole area should reflect the overall anisotropy, and the inside heights should exhibit similar statistics as the outside of the hole area. The whole procedure is illustrated in $\operatorname{ESM}(\mathrm{ESM} 0)$.

Step 1: Laplace healing. Step 1 is necessary to compute the surface height AutoCorrelation Function, ACF, because the surface patch is smooth enough to not disrupt the anisotropy characteristics.

Step 2: textural removal. Step 2 must be tuned regarding the surface correlation lengths: the polynomial degree to be removed should be adjusted to the surface wavelengths. A fifth - or less - order polynomial $P_{5}(x, y)$ leads to satisfactory results with the surfaces used in the present work.

Step 3: surface reconstruction. The principle developed by $\mathrm{Hu}$ and Tonder [11] is recalled hereafter, with their own notation.

Let $\eta$ be the initial heights of the surface, $z$ the desired final heights with the right anisotropy and $h$ the digital filter so that $z=h * \eta$.

Let us suppose that $\eta$ is random white noise. Applying a Fast Fourier Transform, FFT, to the preceding relationship, one easily obtains $Z=H \times A . Z^{2}=H^{2} A^{2}, Z^{2}$ being the $F F T$ of the ACF of $z, Z^{2}=F F T(A C F(z))$ - Wiener-Khinchin theorem.

$A^{2}$ is a constant because $A C F(\eta)=0$ everywhere except in $(0,0) . \quad h$ being symmetric regarding $x$ and $y$, the following relationship holds:

$$
h=F F T^{-1} \sqrt{F F T(A C F(z))}
$$

Hence, providing the height autocorrelation - the surface anisotropy - the digital filter $h$ is determined. The strong assumption here is to consider the surface heights as a random distribution, and more specifically as white noise. What are the consequences? Actually, when applying the digital filter $h$, the resulting heights do not exhibit the 
exact anisotropy as planned, but an approximated one. However this is not a big deal because the aim of the maneuver is to generate a realistic anisotropy inside the hole. Hence having an approximate one is enough.

Step 4: change the heights inside the hole area. After applying the digital filter to the surface heights, the resulting anisotropy is close to the overall anisotropy but the heights are far from being realistic. The idea as exposed by Francisco and Brunetière [12] is to keep the filtered height order but to replace them by more realistic ones. The later are taken in a crown around the hole; if there are not enough heights then, the crown height profile is used to generate the missing heights as illustrated in figure 6.

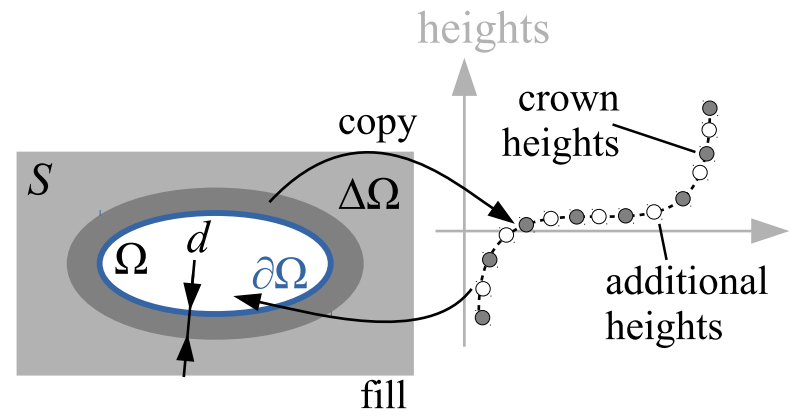

Figure 6: The crown $\Delta \Omega$ heights may not be enough to fill $\Omega$. Therefore the height profile is determined in order to generate additional heights. $S=\Omega \cup \bar{\Omega}$, hole border: $\partial \Omega$

Step 5: reintroduce the macro texture. The last step is the addition of the least square polynomial $P_{5}(x, y)$. The surface heights have been altered outside $\Omega$, so the final surface is the modified $\Omega$ heights combined with the outside hole $\bar{\Omega}$ initial heights.

\subsection{Point-based inpainting}

For each new point inside $\Omega$, the neighboring existing points are examined to find similar zones in $\bar{\Omega}$. When a good candidate is found, the point is copied from $\bar{\Omega}$ into $\Omega$. The procedure, based on Efros' work [?] and Harrison's work [7], is iteratively executed as follows:

Step 1: Initialization

Step 2: Get $\Omega$ and $\partial \Omega$, the unfilled points if any, or terminate

Step 3: For a maximum of ten of unfilled points

- find the best zone to search for correspondences in $\bar{\Omega}$

- find the best patch in the search zone and copy the candidate into $\Omega$

Step 4: return to Step 2 
Step 1: initialization. Before the procedure starts, the anisotropy ratio is determined. In the present work, it is defined as the ratio Stri of the highest correlation length over the smallest. The correlation length is defined as the length where the $A C F$ is $50 \%$ of its maximal value.

If we suppose that what influences a point is its neighbors \pm 3 points - see the figure 7 - and that the closest points influence more the central point that the far ones, then the weighting function can be chosen Gaussian with a standard deviation equal to 6 .

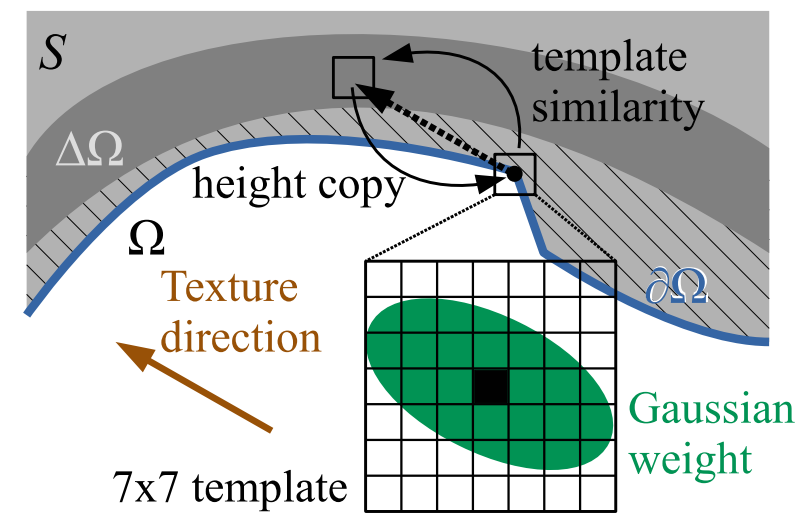

Figure 7: The procedure looks for a similar template in the search zone $\Delta \Omega$ in the direction of the anisotropy. The template points are not given the same weight: it is a 2D Gaussian function which big axis is the anisotropy direction. Once a similar template is found, the central point height is copied into $\partial \Omega$ to fill the hole. The dashed zone is the zone already filled in.

Step 2: get or update $\Omega$. Each unknown point of $\Omega$ must be determined. However, as the process is a point-by-point inpainting process, only the border $\partial \Omega$ points are needed. Depending on how the $\partial \Omega$ point list is determined, the final result can be different. That is why the points are assigned a random order.

Step 3: find a similar template in $\Delta \Omega$ and fill $\partial \Omega$. If the whole border $\partial \Omega$ is filled in one single step, the final result exhibits a kind of connection line as shown on figure 8. Thus, a maximum of ten points are filled in a single step. The surface may exhibit scratches that should be preserved while inpainting $\Omega$. Therefore the first place to look for similarities in $\Delta \Omega$ is in the direction of the anisotropy. The distance $D$ - in other words, the difference - between a $\partial \Omega$-centered $7 \times 7$ kernel and a $\Delta \Omega 7 \times 7$ kernel, is the weighted sum of the height absolute differences. All distances $D$ are calculated, and the $\Delta \Omega$ template that yields the lowest $D$ is determined. The step ends with the copy of the new height - the template central point - into the unknown point. 


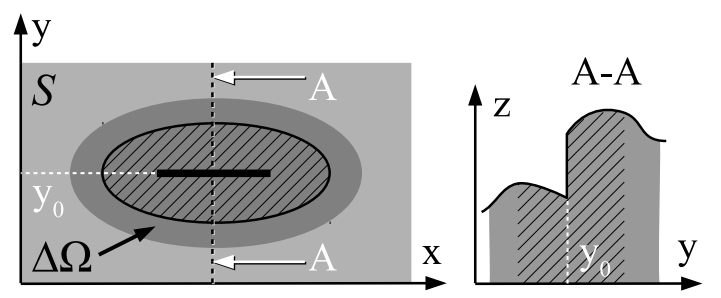

Figure 8: If each new $\partial \Omega$ is filled in one single step, the iterative borders evolve towards the hole center. When the center is reached, a gap is likely to appear between the bottom line and the top line.

Step 4: loop to fill in the remaining points.

\subsection{Exemplar-based inpainting}

The procedure is based on Criminisi's work [8], the general principle is the same as the point-based inpainting. The differences are mainly the copy of the whole template found in $\Delta \Omega$ and the order of filling in $\partial \Omega$. To avoid the patches from being too marked on the final result, leaving a tiled appearance on the repaired hole, the process is carried out four times with smaller and smaller templates.

Step 1: Get $\Omega$ and $\partial \Omega$, the unfilled points if any, or terminate. For each point a priority is assigned.

Step 2: For the point of maximum priority, fill in the empty points of the template with the source template points.

Step 3: return to Step 1, three times, with a smaller template width

The only step that is worth detailing is the first one. Features like scratches make the height gradient $\boldsymbol{\nabla} \boldsymbol{h}$ locally high, it is therefore a means to detect them. In order to extend the scratches from $\partial \Omega$ into $\Omega$ in the right direction, $\nabla \partial \Omega$ is determined, and if $\boldsymbol{\nabla} \boldsymbol{\partial} \boldsymbol{\Omega} . \boldsymbol{\nabla} \boldsymbol{h}=0$ at a given point $P$, it means that the hole frontier is perpendicular to the scratch: $P$ must be firstly filled in. The figure 9 shows a situation where points have already been filled in - dashed zone - and a priority $p$ is assigned to point $P$ : $p=\boldsymbol{\nabla} \partial \boldsymbol{\Omega} \cdot \boldsymbol{\nabla}^{\perp} \boldsymbol{h}$. Large values of $p$ on $\partial \Omega$ means that the frontier is locally perpendicular to a marked feature. 


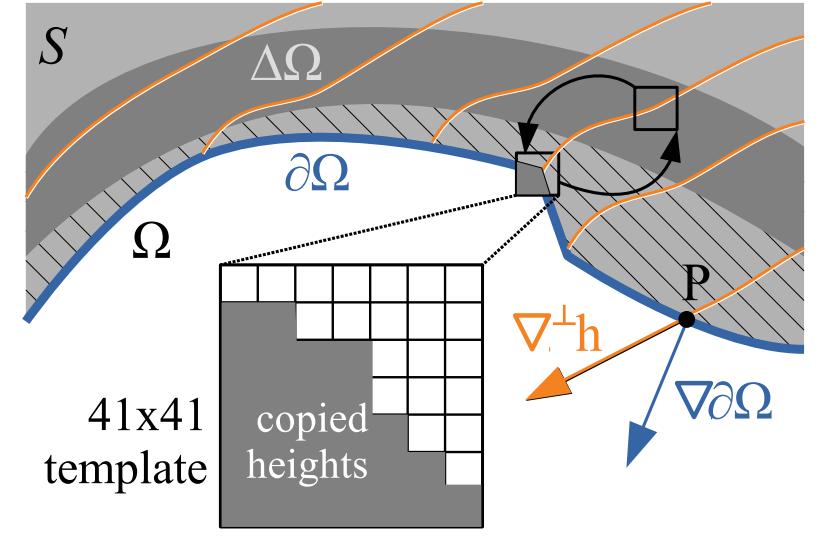

Figure 9: $\partial \Omega$ is not randomly filled. To preserve the texture, the height gradient $\boldsymbol{\nabla} \boldsymbol{h}$ is determined on $\partial \Omega$. At point $P$, if $|\boldsymbol{\nabla} \boldsymbol{h}|$ is important and if the normal to $\partial \Omega$ is colinear to $\nabla^{\perp} \boldsymbol{h}$, it means that $P$ is in the continuity of a relief element, like a scratch, and then is assigned a higher priority.

\subsection{Surface parameters}

The parameters that are tested on the Anisotropy1-2-3 surfaces are detailed by Francisco et al. [9], [10]. The parameters can be classified as spatial or anisotropy parameters, height parameters, topology parameters and fractal parameters. Why investigating the effect of surface healing on many different parameters? Provided the fact that the healed surface is to be analyzed, the recreated zones should alter as less as possible the results of the same sane surface: the new patch should meet the anisotropy conditions, the height conditions and the micro-texture conditions of the sane surface.

3.5.1. 3 spatial parameters. Spatial parameters are related to the height ordering. According the way that heights are organized, longer or shorter wavelengths appear. The anisotropy of a surface is related to the presence of preferred texture directions, then, a means to assess the anisotropy is to determined the AutoCorrelation Function, ACF. A detailed presentation is given by Francisco et al. [9].

On a normalized $A C F$, an horizontal cutting plane at height $0<s<1$ defines an ellipsis-like line. Defining the minor and major ellipsis radii as Rmax and Sal respectively, the higher Stri=Rmax/Sal the more anisotropic surface. In the present work $s=0.5$ and because Stri, Sal and Rmax are linked, Sal is left aside.

Another way of catching anisotropy is to study the ratio r.sl of the maximum of the $A C F$ slope to the minimum: it quantifies the $A C F$ relative decay speed in nearly perpendicular directions.

\subsubsection{3 height parameters. Height parameters mainly refer to height statistics.}

High order statistical moments are sensitive to outliers so, the height skewness Ssk and kurtosis $S k u$ are chosen; if the healing process introduces abnormal heights or an 
abnormal height distribution, $S s k$ and $S k u$ are affected.

An additional height parameter is analyzed, the height mean $S m$. Although it is not a relevant parameter for the whole surface, when the surface is sampled, it becomes a wear marker. For instance, when dealing with dental surfaces, it reveals to be a diet discriminative parameter associated to locally stepped surfaces [10].

3.5.3. 2 topological parameters. Topological parameters are defined as parameters determined by morphological operations, such as binarization, dilation, erosion, etc., on surfaces.

Sh is the percentage of quasi-horizontal faces - face normal within a four degrees cone. It is sensitive to the surface long wavelength relief.

The ratio area $S k 1$ is the fraction of the surface heights above $85 \%$ of the height amplitude, it is sensitive to high elevation zones.

3.5.4. 3 fractal parameters. Fractal parameters are defined as parameters that take into account the surface area at different scales.

The relative area $S d a r$ - developed area/projected area - strongly depends on the fractal dimension of the surface: the greater fractal dimension, the higher relative area.

asfc is called "surface complexity", and it is defined as a thousand times minus the steepest slope of the function $\log (S d a r)=f(\log (s e))$, with se the element size at a given grid scale, see [10] for further details.

indf is a fractal index calculated with the box-counting method. It is the slope of the function $\log (n b)=f(\log (s e))$, where $n b$ is the number of boxes of size se under the surface at a given scale.

3.5.5. Surface sampling. As the figures 1,2,3 suggest, the surfaces are not homogeneous. The key idea, as exposed by Francisco et al. [9], [10], is to uniformly sample the surface. Different tries have been carried out to determine an optimal number of samples, but experience has proved that it is very parameter-dependent. Indeed, when one tries to separate several diet categories upon a set of parameters, the different numbers of samples make the groups overlap more or less: in the end, it is all about compromise, the samples should not be too small or too large. In the present work, a $16 \times 16$ image slicing is chosen with $256 \times 256$ pixels sub-surfaces.

Several statistics are built from the sub-surfaces parameters:

- min.05 the parameter mean of the first $5 \%$ values, it is a kind of robust minimum,

- fst.25 the parameter first quartile,

- med the parameter median value,

- lst.25 the parameter third quartile, and

- max.05 the parameter mean of the last 5\% values, it is a kind of robust maximum.

Hence, to each parameter calculated on the whole surface - suffixed with _glob - 5 derived parameters are added. 


\subsection{Analysis procedure}

Two different but complementary studies are carried out to answer the questions: what is the effect of the damaged area size on the healing quality? What is the effect of the damaged area location on the healing quality?

3.6.1. Healed area size influence. A $1024 \mathrm{px} \times 1024 \mathrm{px}$ sub-surface is extracted from each of Anisotropy1-2-3 surfaces. The study could as well be carried out on the $1551 \mathrm{px} \times 1551 \mathrm{px}$ original surfaces but extracting a sub-surface allows for centering particular features - the deep scratch of Anisotropy1, figure 11, for instance; the impact of healing is thus better tested because the damaged zone is centered. It should be noted that the anistropy ratio is barely altered.

Six hole sizes are tested for healing. The holes are ellipsis-shaped, as on image processing software when defect area are selected. The table 1 details the hole sizes, as well as $D a$, the ratio of the damaged area on the whole surface area.

\begin{tabular}{ccc}
$a(\mathrm{px})$ & $b(\mathrm{px})$ & relative area $D a(\%)$ \\
\hline 71 & 35 & 0.75 \\
100 & 50 & 1.50 \\
141 & 71 & 3.00 \\
200 & 100 & 6.00 \\
283 & 142 & 12.00 \\
400 & 200 & 24.00 \\
\hline
\end{tabular}

Table 1: Six hole sizes are tested to analyze the defect size influence on the healing results. $a$ and $b$ are the semi-major and semi-minor axes respectively.

We investigate here to what extend the defects should be treated with one healing method rather than another. It is expected that whatever the parameter involved, the values on global-, point- or exemplar-healed surfaces, will be closer to the values on the original surface, than for Laplace's infilling. For common sense reasons, the healed surface results should also be better for smaller damaged areas.

Table 2 recaps the whole parameter set; it represents more than 4750 cases. 


\begin{tabular}{|c|c|c|c|c|c|}
\hline Surface & $D a$ & Healing method & \multicolumn{2}{|c|}{ Parameters } & Sampling statistics \\
\hline Anisotropy1 & 0.75 & (E)xemplar & Stri & & glob \\
\hline Anisotropy2 & 1.50 & (G)lobal & $R \max$ & Spatial & $\min .05$ \\
\hline Anisotropy3 & 3.00 & (L)aplacian & r.sl & & fst.25 \\
\hline & 6.00 & (P)oint-based & Ssk & & med \\
\hline & 12.00 & & Sku & Height & lst.25 \\
\hline & 24.00 & & $S m$ & & $\max .05$ \\
\hline & & & $\begin{array}{c}S h \\
S k 1\end{array}$ & Topology & \\
\hline & & & $\begin{array}{l}\text { Sdar } \\
\text { asfc } \\
\text { indf }\end{array}$ & Fractal & \\
\hline
\end{tabular}

Table 2: Recap of the different case combinations that are computed

3.6.2. Statistical analysis. To ensure that the four healing methods yield very different results, the damaged area should be large enough. However too large an area would be unrealistic. The authors have chosen to randomly scatter four non-overlapping holes for an amount of $2.6 \%$, figure 10. As it will be seen with the first analysis, $D a=3 \%$ is enough for ranking the healing methods. However, a single $3 \%$ defect is not accepted in common use, because even if the ratio seems low enough, it is considered too much intrusive. It is the reason why we choose to break the "large" defect into four parts because an experienced user is more likely to accept several smaller defects rather than a large one. In addition, four elements let more location possibilities than a single one.

Then, the healing processes are carried out and the parameters are calculated for comparison with the original surface.



Figure 10: $1551 \times 1551$ surface with four elliptic holes covering $2.6 \%$ of the surface area. The same surface ratio is presented in a single elliptic shape in the bottom left. A $256 \times 256$ sample is also represented. 
The hole locations are not equivalent regarding the healing results, that is the reason why the calculations are performed many times. In order to compare the results using statistical tests, the amount of calculations must be high enough: this way, the tests will be more robust against departure from normality of the result distributions. $n=512$ - the number of runs carried out for each case - is found to be a good compromise between statistical significance and computing time.

When performing a statistical test, an hypothesis $H_{0}$ is assumed. As an example, to test the normality of a set of data, one can use the well-known Shapiro-Wilk test; $H_{0}$ is "the data are normally distributed". For the moment, let us put aside the test assumption requirements. Some results, that will be presented in the result section, illustrate the whole set of $n$ values for a given parameter, on a given surface, for each inpainting method, as well as the parameter value on the original surface. Let us suppose that an inpainting method has a mean value closer to the original one than another inpainting method, but that it has a greater variance in the mean time. Which one of the two is the best choice to fill in the holes? This is where the statistical tests can help. In particular, the one-sample Student $t$-test explores the possibility for a set of data to have a given value $\mu_{0}$ as mean value. In the present study, the set of data is the $n$ runs of four holes randomly scattered on a surface, and $\mu_{0}$ is the parameter value calculated on the original surface.

One of the outputs of the statistical test is the well-known p-value, which is the probability to find sets of data more extreme that the one studied, assuming that $H_{0}$ is true, i.e. that the set mean value is $\mu_{0}$. In other words, the p-value quantifies how well the data fit $H_{0}$. The p-value is a controversial subject because it is sometimes interpreted as how much the theory $H_{0}$ is truthfully; however, it is used here to compare the data distributions to a given mean value $\mu_{0}$, assuming $H_{0}$.

As stated before, a statistical test requires more or less assumptions. The Student $t$-test assumes that the data are independent, normally distributed with no outlier. In a more practical way, as the $t$-test is fairly robust against departures from normality, especially in large samples [13], we seek for near-normality and the $2 \%$ extreme values are excluded from the sample. It is to be noted that arbitrarily removing data from the data set is not recommended by some authors, as Quinn [14] p.68: [...] dropping observations just because they are messy or reduce the chance of getting a significant result is unethical, to say the least. The authors of the present study agree this statement; however here, potential outlier data are not chosen but rather systematically dropped.

For maximal chances to get near-normality, the data can be first monotonously transformed. The Box-Cox transformation is a mathematical function that is automatically tuned to make the data closer to normality as possible. Even if it is of low interest because the data set is large - almost 500 values - the trick is anyway applied. In some cases the result distributions are too much skewed to just ignore it.

To end with the analysis protocol, as some results exhibit multi-modality, an additional statistical test is used. The one-sample Wilcoxon rank test is a non- 
parametric test, sometimes described as the alternative to the Student $t$-test when the data cannot be assumed to be normally distributed. This time the data median value is compared to the original value. The data being ranked, there is no need for normality but just symmetry. Once again with so large a data set, reasonable departures from perfect symmetry are allowed.

It is expected here that one of global-, point- or exemplar-healing turns to be a statistically much better choice than Laplace's because the results are visually far more realistic.

\section{Results and discussion}

\subsection{Healed area size influence}

4.1.1. Visual results. The following figures - from 11 to 13 - show the infilling results on the three surfaces Anisotropy1-2-3, with a central hole; the hole relative area is $D a=12 \%$.

Laplace-based method seems to visually yield very poor results, while the other methods create realistic features. The results are quite impressive but to what extend the reconstructed area can be trusted? To put it simple, can we accurately replace any real area by a numeric patch? The answer is intuitively no, and it can be numerically brought to light with surface parameter comparisons. 



Figure 11: The original surface (O) - Anisotropy1 - is shown in central position. An elliptic area is erased on the surface center, and four infilling methods are tested: Laplace (L) top left, for which the infilled area clearly appears, Global-based $(\mathrm{G})$ top right, Point-based (P) bottom left and Exemplar-based (E) bottom right 

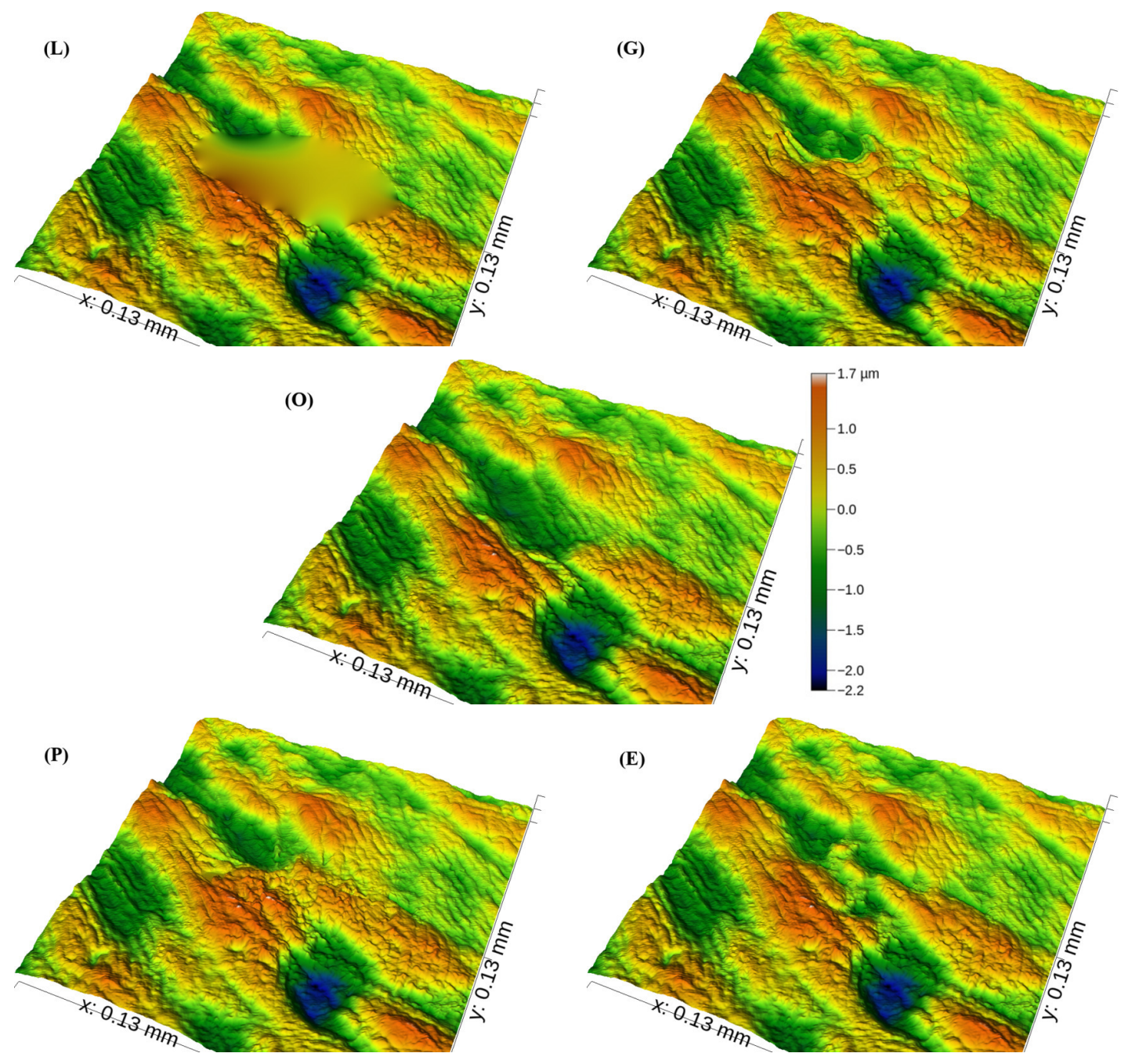

Figure 12: The original surface (O) - Anisotropy2 - is shown in central position. An elliptic area is erased on the surface center, and four infilling methods are tested: Laplace (L) top left, for which the infilled area clearly appears, Global-based $(\mathrm{G})$ top right, Point-based (P) bottom left and Exemplar-based (E) bottom right 

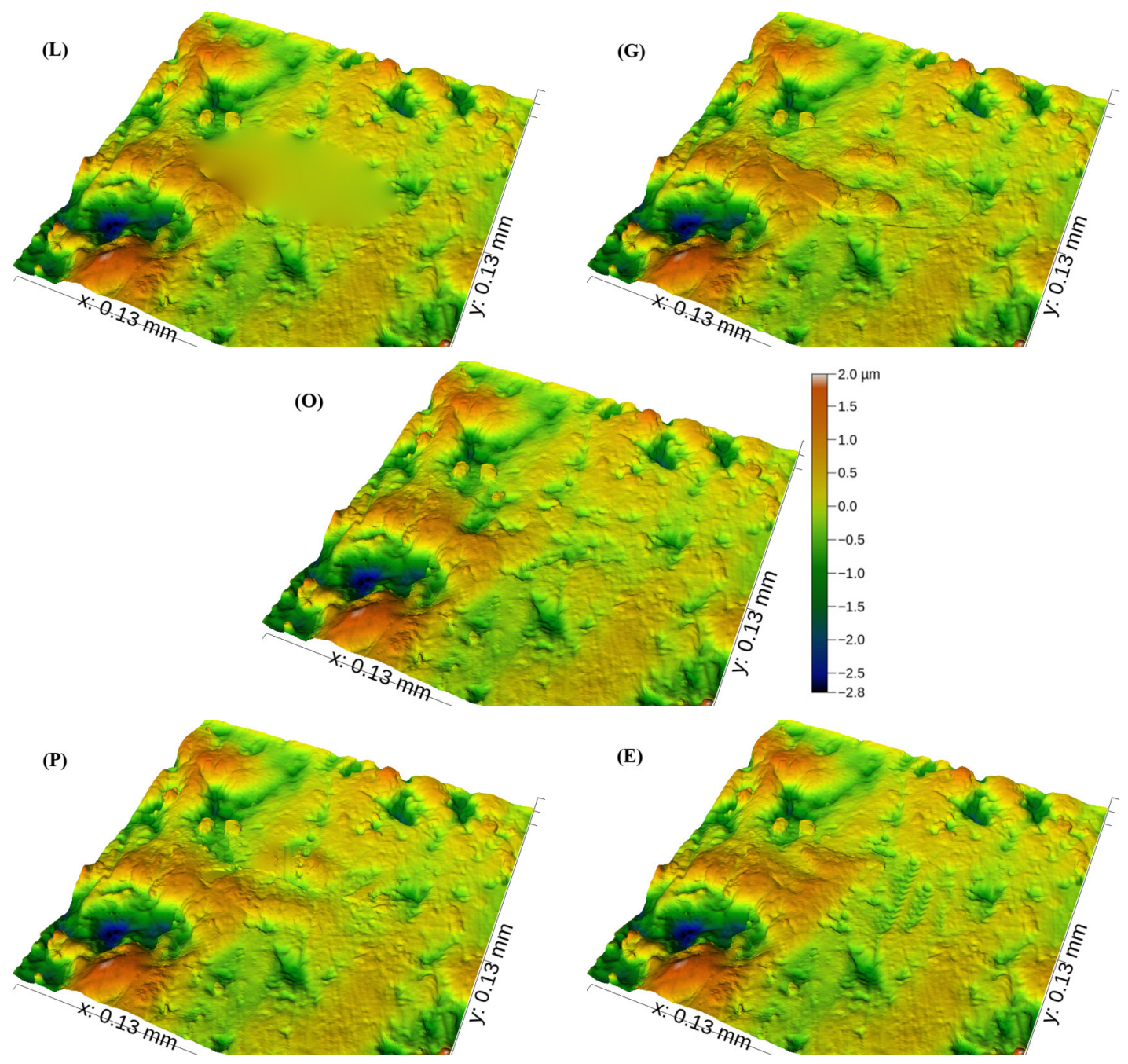

Figure 13: The original surface (O) - Anisotropy3 - is shown in central position. An elliptic area is erased on the surface center, and four infilling methods are tested: Laplace (L) top left, for which the infilled area clearly appears, Global-based (G) top right, Point-based (P) bottom left and Exemplar-based (E) bottom right

4.1.2. Quantitative results by surface. There are too many graphs to present them all, see Table 2. Hereafter - figure 14 - are some representative cases of the parameters calculated on the whole surface Anisotropy1: each plot synthesizes the results of a single parameter calculated on a whole particular surface, for the different hole sizes and the different methods. We choose to illustrate the results on the whole surface rather than a particular statistics (min.05, fst.25, med, lst.25, max.05) to help the understanding. 

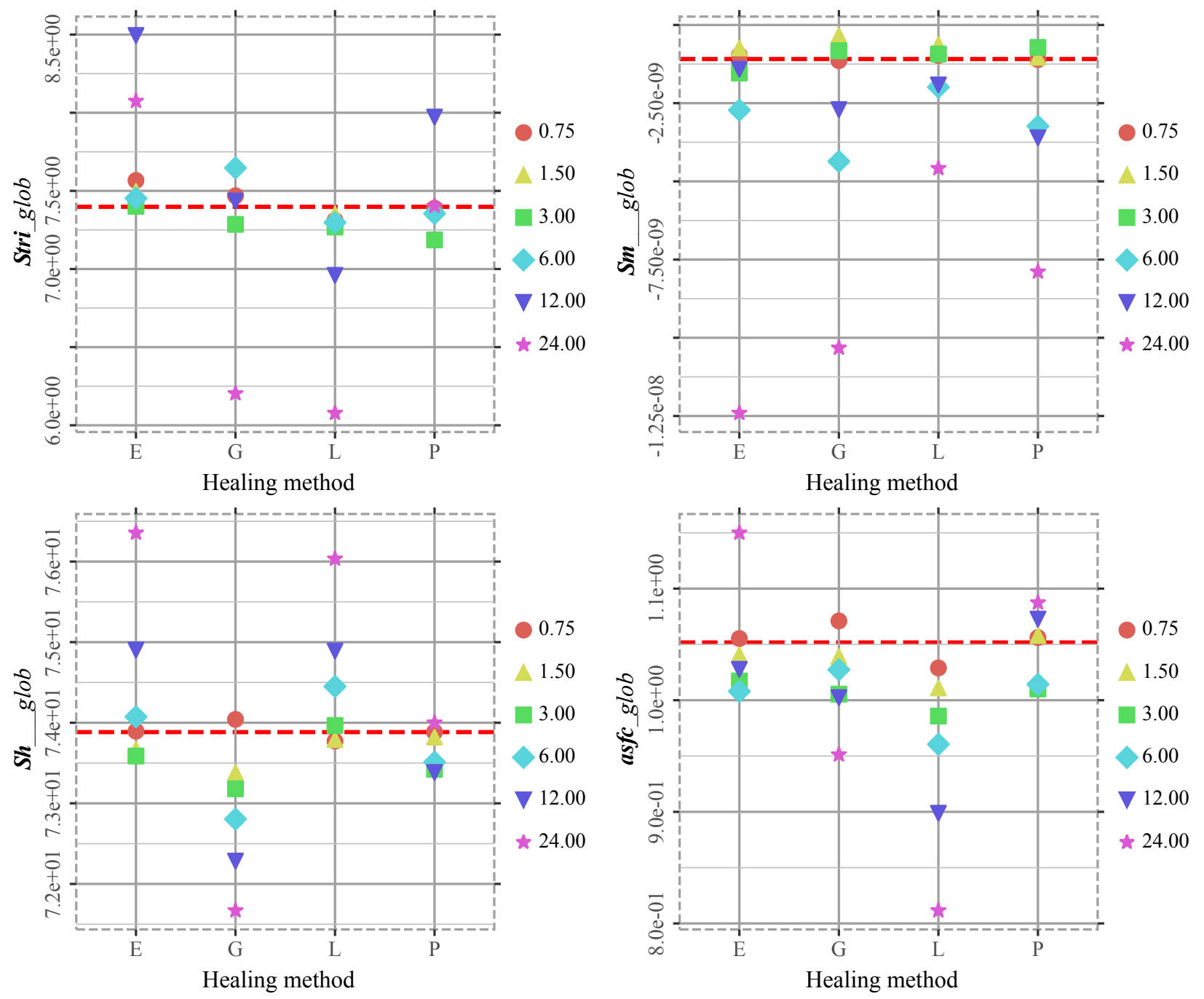

Figure 14: Four different category parameters calculated on the surface Anisotropy1 are presented. Top left, the anisotropy factor Stri; top right, the mean height Sm; bottom left, the horizontality ratio $S h$; bottom right the area scale factor asfc.

The healing methods are: (E) the exemplar-based method; $(\mathrm{G})$ the global method; $(\mathrm{L})$ Laplace's method; (P) the point-based method.

The different hole relative sizes are expressed as percentages.

In $\mathrm{ESM}(\mathrm{ESM} 1)$ almost all remaining parameters are presented on the same surface, Anisotropy1.

As it clearly appears on the figure 14, the largest damaged areas - Da $\geqslant 12 \%-$ yield the poorest results. It was nonetheless foreseeable that an artificial process could not reproduce such an important patch, despite visually convincing results.

The anisotropy factor Stri is not more than 3.5\% different from the theoretical value if $D a \leqslant 6 \%$. Laplace's operator shows rather good results, which could appear surprising at first sight, but as it can be seen on figure 11, it introduces no local parasitic anisotropy. Therefore, the overall anisotropy remains well caught by the autocorrelation 
function.

The mean height $S m$ seems to suffer more from surface healing, but a look at the scale reveals very small values, the variation remaining within a few nanometers. Once discarded the $D a \geqslant 12 \%$ restoration cases, the healing processes do not alter much $S m$, Laplace's being the best choice. It is simply explained by the fact that the theoretical patch has a mean value very close to the whole surface; thus, Laplace's smooth patch barely modifies it.

The flatness ration $S h$ is well preserved with point- and exemplar-based methods, the error being around $0.8 \%$ when $D a \leqslant 6 \%$. This is due to the method main characteristic: the reproduction of the neighboring relief. As concerns Laplace's method efficiency here, the reason is different. The patch being already almost $74 \%$ horizontal, Laplace's operator doesn't alter much Sh. In ESM(ESM2), the reader can find:

- the results of the figure 14 cleared from the two cases $D a \geqslant 12 \%$,

- the results for the two other surfaces Anisotropy2-3 showing lower global flatness, and poorer results for Laplace's method.

It is out of the question to choose in practice the healing method for a given analysis because:

- there are often different kinds of parameters associated to a surface study,

- the methods are always improvable, except for Laplace's which is a particular equation implementation,

- the quality of the results depends obviously on the surface features, on their homogeneity, etc.

Here however, we can rank the methods upon their ability to recreate parts of a surface by averaging the results:

- on the three surfaces Anisotropy1-2-3,

- with all $D a$ values,

- of all sub-parameters.

The reader is referred to ESM(ESM3) for a short explanation on the ranking process.

Doing so, the ranks shown on figure 15 highlight the advantages in using a pointbased technique to heal damaged surfaces. As warned before, restoring images with $D a \geqslant 12 \%$ is not realistic but, if the two related cases - $12 \%$ and $24 \%$ - are excluded from the results, the graphs remain approximately the same. 

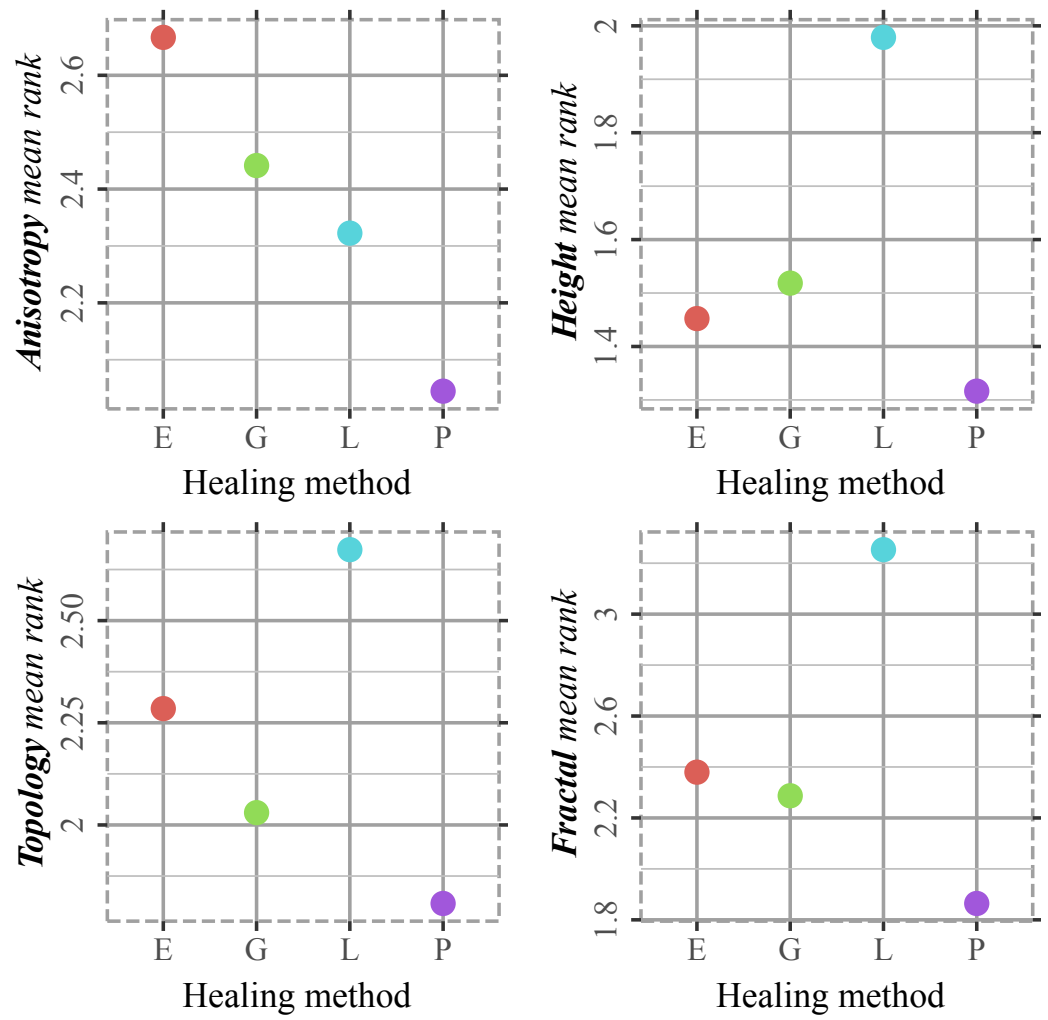

Figure 15: The four ranked categories are presented. Top left, the anisotropy category; top right, the height category; bottom left, the topology category; bottom right the fractal category.

The healing methods are: (E) the exemplar-based method; $(\mathrm{G})$ the global method; $(\mathrm{L})$ Laplace's method; (P) the point-based method. The lower rank, the better performance.

Additional results, that detail the synthetic results, are also provided in $\operatorname{ESM}(\mathrm{ESM} 3)$.

\subsection{Defect location influence}

When surfaces are not perfectly homogeneous, the damaged area locations are not equivalent from a healing point of view. In addition, as exposed in the method section, several moderate size damaged areas are more likely to be accepted than a single important one. The reasons for multiple defects to occur are

- the real surface is not clean enough: there are remaining debris on the surface

- the microscope light is locally badly reflected: when the surface exhibits very steeped faces, the reflected rays are not caught

- the surface mold has trapped air bubbles, etc.

The figure 16 synthesizes the results of the 512 runs carried out on the surface Anisotropy1 for the same parameters as in the previous section, and a damaged area 
$D a=2.6 \%$. See ESM(ESM4) for Anisotropy2-3. The first observation to be made is the pretty good symmetry of the data which safely allows one-sample Wilcoxon rank tests. Even if the data do not follow a normal law, thanks to the sample number and the low skew coefficient it is also expected Shapiro-Wilk tests to be applicable.

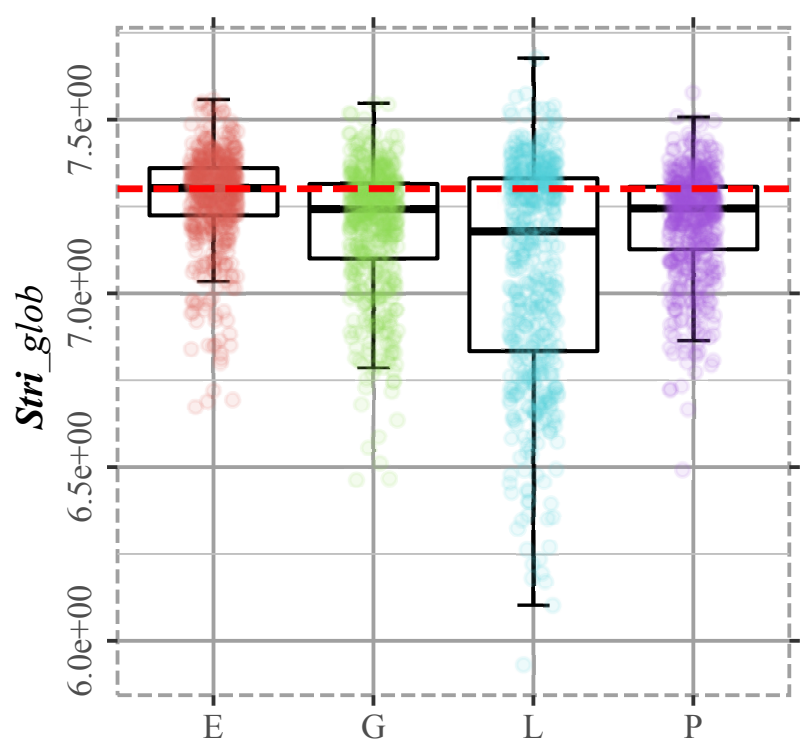

Healing method

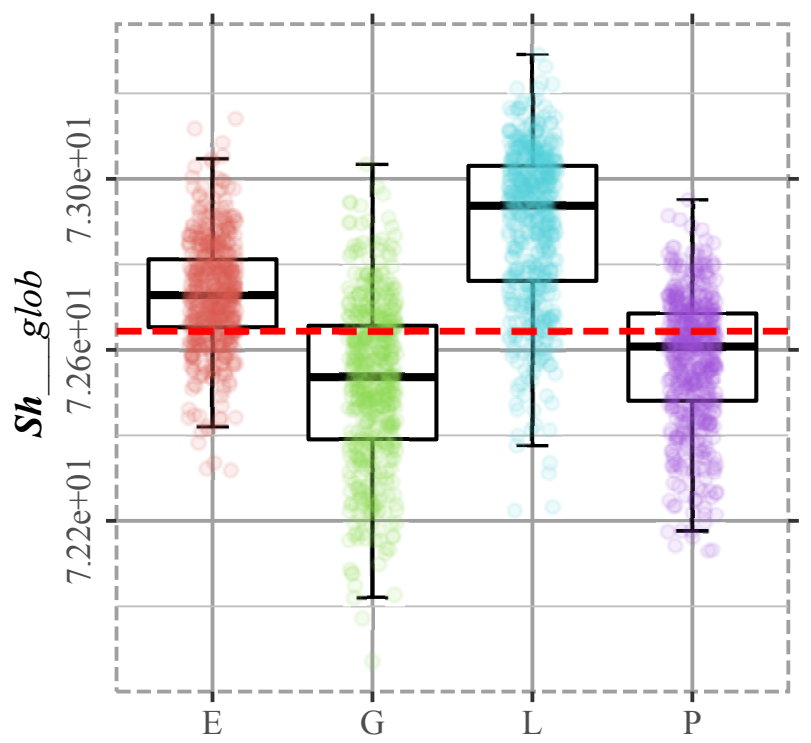

Healing method



Healing method

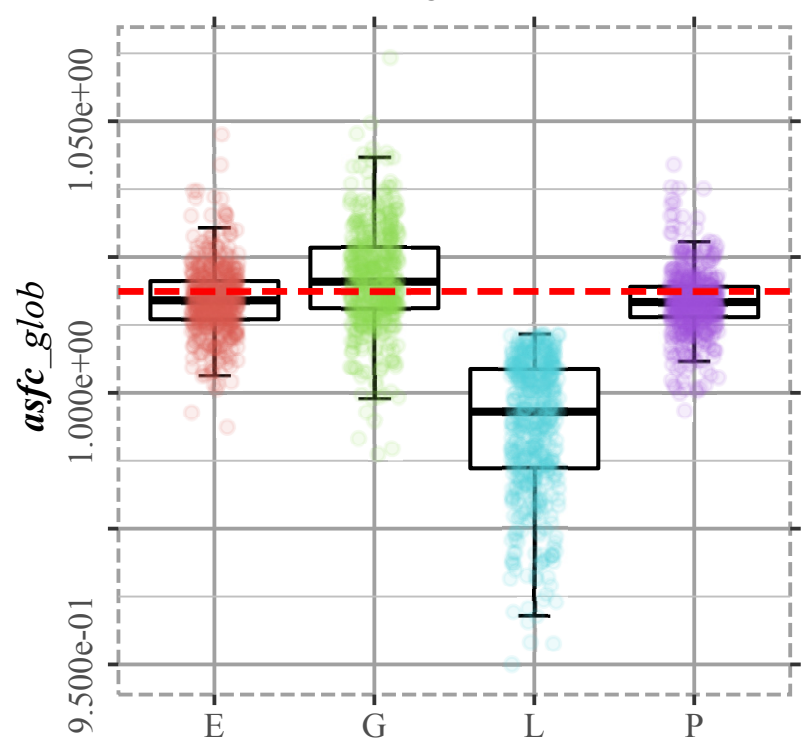

Healing method

Figure 16: Four different category parameters calculated on the surface Anisotropy1 are presented $(D a=2.6 \%)$. Each circle is a random location of the holes. Top left, the anisotropy factor Stri; top right, the mean height $S m$; bottom left, the horizontality ratio $S h$; bottom right the area scale factor asfc.

The healing methods are: (E) the exemplar-based method; $(\mathrm{G})$ the global method; (L) Laplace's method; $(\mathrm{P})$ the point-based method. 
Depending on the location of the damaged areas, Laplace's operator can significantly alter the anisotropy factor Stri: the range of values is at least twice than for the other methods. As a consequence, there are many points with a $10 \%$ error, even though the mean value remains close to the theoretical value. This statistical study gives clearly advantage to the exemplar-based method which contradicts the synthetic results of the figure 15. But, having a closer look on the more detailed results, figure 14 with $D a=3 \%$, the exemplar-based method can provide the theoretical value. Actually, there is no real contradiction here: the mean rank results take into account the two other anisotropy parameters as well as their sub-parameters, i.e. fst.25, min.05, etc. Moreover, in the previous section, the damaged area was centered so the conclusions must remain cautious. The global method, which aimed to be particularly suited for anisotropic surfaces, yields at the end a bit disappointing results.

Concerning Sm results - the height mean - Laplace's patches approximate well the real areas. The same explanations as in the previous section hold. The two other parameters, the topological parameter $S h$ and the fractal parameter asfc, have less close values to the right values with Laplace's method than with other methods.

Figure 17 proves the meaningfulness of the statistical tests: despite some minor differences, the parametric test (Shapiro-Wilk) and non-parametric test (Wilcoxon) give the same results. The exemplar-based method - except for the height parameters - and the point-based method are the best way to heal the damaged surfaces. Once again, this statement does not contradict with the previous section observations because the situation is different. First, in the present situation $D a \simeq 3 \%$ and it is summed over four areas, second, figure 17 is based on statistics; therefore many different cases are averaged, unlike the previous single cases. The reader may also refer to ESM(ESM5) for less synthetic results. 

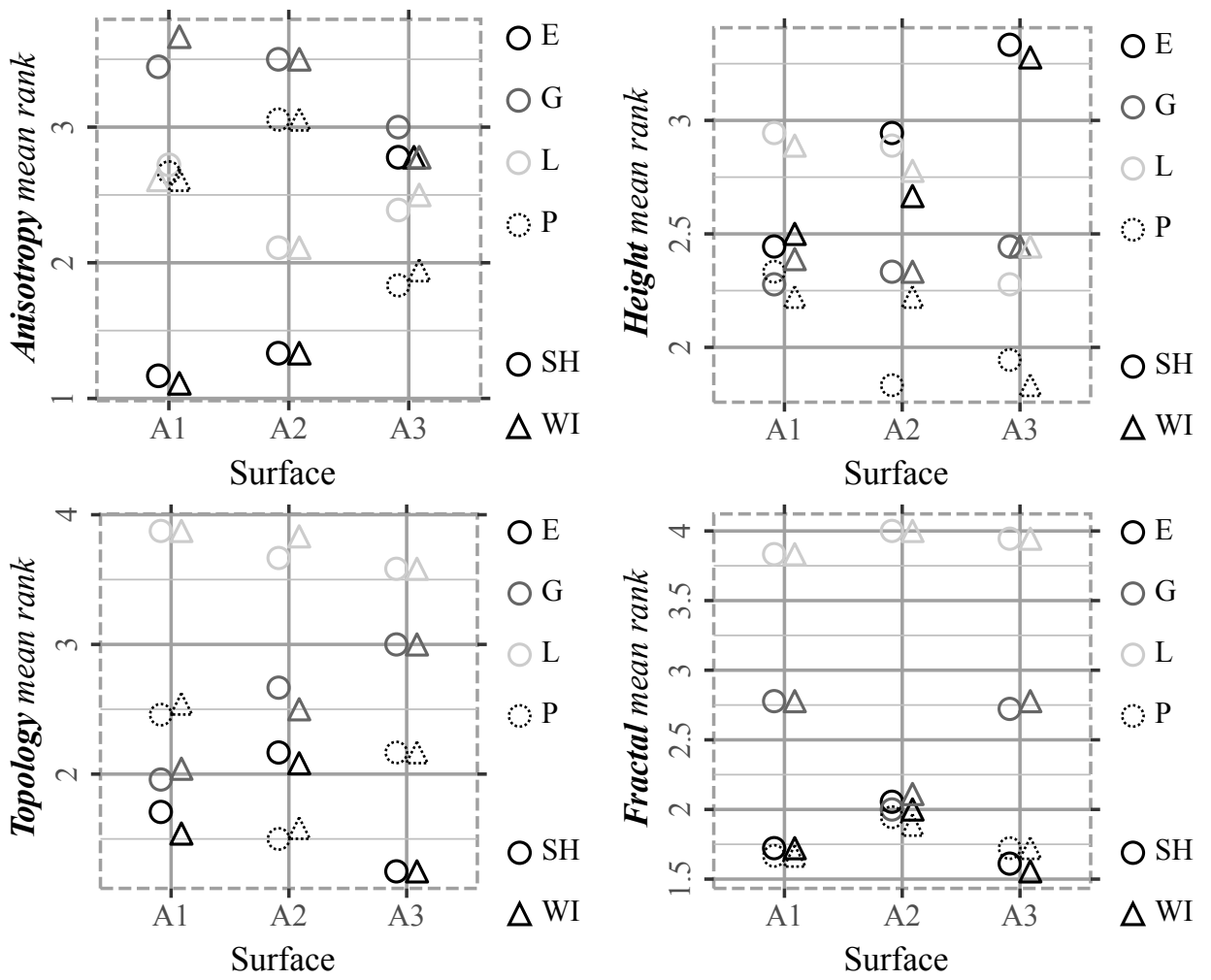

Figure 17: The four ranked categories are presented. Top left, the anisotropy category; top right, the height category; bottom left, the topology category; bottom right the fractal category.

The healing methods are: (E) the exemplar-based method; $(\mathrm{G})$ the global method; (L) Laplace's method; (P) the point-based method. Two statistical tests are used: parametric (SH) and non-parametric (WI)

When averaging the results on the surfaces Anisotropy1-2-3 and the two tests, the inpainting methods can be ranked, as proposed on figure 18. The conclusion is straightforward because the graphs show the best performance for point- and exemplarbased methods. If we take into account the previous section conclusions, it can be safely stated that the visual feelings about the different process quality are correlated with the quantitative analyses. Let us however remain careful about the superiority of the methods. The rank scales are not the same for the different parameter categories. The ranks are somewhat close to each other in the height graph, which means that Laplace yields not so much different results than the other methods. 

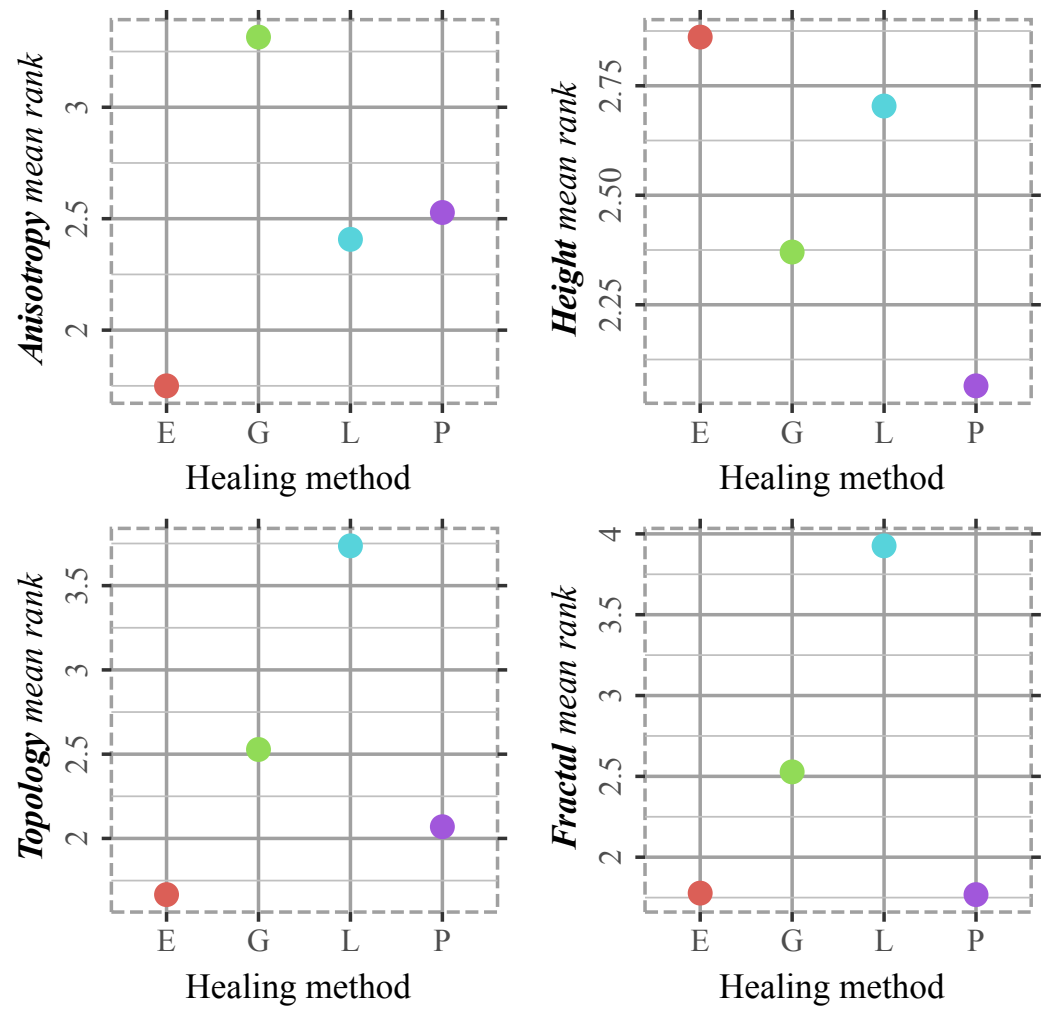

Figure 18: The four ranked categories are presented. Top left, the anisotropy category; top right, the height category; bottom left, the topology category; bottom right the fractal category.

The healing methods are: (E) the exemplar-based method; $(\mathrm{G})$ the global method; $(\mathrm{L})$ Laplace's method; (P) the point-based method.

\section{Conclusion}

The concluding remarks are the following:

- From a visual point of view, Laplace's results are the worst

- Despite very convincing visual aspects, except for Laplace, the healing processes significantly alter the results when the damaged area exceeds $3 \%$

- Developing a method to specifically handle anisotropic surfaces turns not to be useful, compared to common methods like point- and exemplar-based methods

- Two categories of parameters are particularly sensitive to Laplace's inpainting: topology and fractal

- Statistics show that point- and exemplar-based methods produce better results than Laplace's method.

Point- and exemplar-based methods have been developed twenty years ago and are still improvable. They are fast, but for digital surface healing, speed is not a key point, accuracy is. We recognize that for scattered unread points - e.g. because of 
high local slopes - Laplace remains well suited. But, we think that it is worth testing more advanced methods like wavelet decomposition methods, or more recent ones like AI-based methods, when large damaged area occur. Otherwise, for moderate damaged areas, point- and exemplar-based methods remain efficient tools. 


\section{References}

[1] Guilin Liu, Fitsum A. Reda, Kevin J. Shih, Ting-Chun Wang, Andrew Tao, and Bryan Catanzaro. Image Inpainting for Irregular Holes Using Partial Convolutions. In Vittorio Ferrari, Martial Hebert, Cristian Sminchisescu, and Yair Weiss, editors, Computer Vision - ECCV 2018, Lecture Notes in Computer Science, pages 89-105, Cham, 2018. Springer International Publishing.

[2] Paul Francis Harrison. Image Texture Tools. Diss. PhD thesis, Monash University, 2005.

[3] Shengrong Gong, Chunping Liu, Yi Ji, Baojiang Zhong, Yonggang Li, and Husheng Dong. Advanced Image and Video Processing Using MATLAB. Modeling and Optimization in Science and Technologies. Springer International Publishing, 2019.

[4] Tudor Barbu. Novel Diffusion-Based Models for Image Restoration and Interpolation. Signals and Communication Technology. Springer International Publishing, 2019.

[5] Marcelo Bertalmio, Guillermo Sapiro, Vincent Caselles, and Coloma Ballester. Image Inpainting. In Proceedings of the 27th Annual Conference on Computer Graphics and Interactive Techniques, SIGGRAPH '00, pages 417-424, New York, NY, USA, 2000. ACM Press/Addison-Wesley Publishing Co.

[6] M. Bertalmio, L. Vese, G. Sapiro, and S. Osher. Simultaneous structure and texture image inpainting. IEEE Transactions on Image Processing, 12(8):882-889, August 2003.

[7] Paul Harrison. A non-hierarchical procedure for re-synthesis of complex textures. In Proceedings of The 9-Th International Conference in Central Europe on Computer Graphics, Visualization and Computer Vision'2001, pages 190-197. University of West Bohemia, 2001.

[8] A. Criminisi, P. Perez, and K. Toyama. Region filling and object removal by exemplar-based image inpainting. IEEE Transactions on Image Processing, 13(9):1200-1212, September 2004.

[9] Arthur Francisco, Cécile Blondel, Noël Brunetière, Anusha Ramdarshan, and Gildas Merceron. Enamel surface topography analysis for diet discrimination. A methodology to enhance and select discriminative parameters. Surface Topography: Metrology and Properties, 6(1):015002, 2018.

[10] Arthur Francisco, Noël Brunetière, and Gildas Merceron. Gathering and Analyzing Surface Parameters for Diet Identification Purposes. Technologies, 6(3):75, August 2018.

[11] Y. Z. Hu and K. Tonder. Simulation of 3-D random rough surface by 2-D digital filter and fourier analysis. International Journal of Machine Tools and Manufacture, 32(1):83-90, February 1992.

[12] Arthur Francisco and Noël Brunetière. A hybrid method for fast and efficient rough surface generation. Proceedings of the Institution of Mechanical Engineers, Part J: Journal of Engineering Tribology, 230(7):747-768, July 2016.

[13] Peter Dalgaard. Introductory Statistics with R. Statistics and Computing. Springer New York, New York, NY, 2008.

[14] Gerry P. Quinn and Michael J. Keough. Experimental Design and Data Analysis for Biologists. Cambridge University Press, Cambridge, UK ; New York, 1 edition edition, April 2002. 